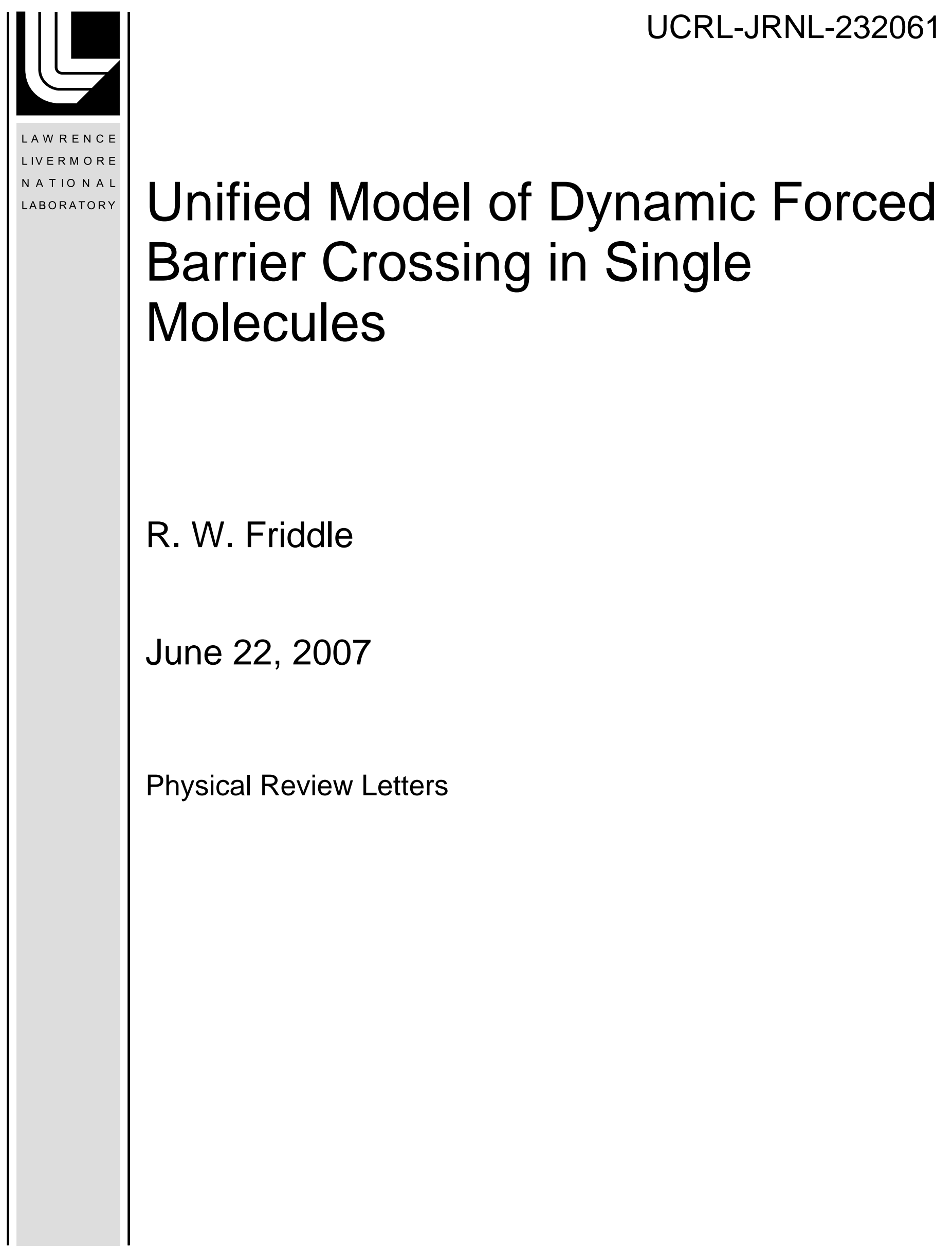


This document was prepared as an account of work sponsored by an agency of the United States government. Neither the United States government nor Lawrence Livermore National Security, LLC, nor any of their employees makes any warranty, expressed or implied, or assumes any legal liability or responsibility for the accuracy, completeness, or usefulness of any information, apparatus, product, or process disclosed, or represents that its use would not infringe privately owned rights. Reference herein to any specific commercial product, process, or service by trade name, trademark, manufacturer, or otherwise does not necessarily constitute or imply its endorsement, recommendation, or favoring by the United States government or Lawrence Livermore National Security, LLC. The views and opinions of authors expressed herein do not necessarily state or reflect those of the United States government or Lawrence Livermore National Security, LLC, and shall not be used for advertising or product endorsement purposes. 


\title{
Unified Model of Dynamic Forced Barrier Crossing in Single Molecules
}

\author{
Raymond W. Friddle \\ Chemistry, Materials, Earth, and Life Sciences Directorate, \\ Lawrence Livermore National Laboratory, Livermore, California 94550, USA
}

\begin{abstract}
Thermally activated barrier crossing in the presence of an increasing load can reveal kinetic rate constants and energy barrier parameters when repeated over a range of loading rates. Here we derive a model of the mean escape force for all relevant loading rates - the complete force spectrum. Two well-known approximations emerge as limiting cases; one of which confirms predictions that singlebarrier spectra should converge to a phenomenological description in the slow loading limit.
\end{abstract}

PACS numbers: 73.22.Dj, 82.37.Np, 87.15.Aa

Continued progress in magnetic [1], optical [2] and mechanical [3] manipulation has enabled experimentalists to study the microscopic states of systems ranging from single molecule magnets to individual biological complexes. A particularly common technique is found by steadily increasing a force field until a change in state is observed (such as a switch in domain polarity [4] or the dissociation of an intermolecular bond [5]). The power of this technique relies on theoretical developments which indicate that repeating such a measurement over a range of loading rates can enable the normally hidden details of the intrinsic energy landscape to be determined [6-8].

The current models of dynamically forced escape are incomplete in that they only describe extreme loading rate regimes. The purpose of this Letter is to present a solution of the mean escape field for all rates of increasing force that permit thermally activated escape. The model is based on the adiabatic evolution of Kramers' escape [9] from a model energy landscape under an increasing load. It is shown that in the slow and fast loading limits two prominent solutions of dynamically forced escape emerge. Throughout this Letter we employ Evans' [6] nomenclature of the "spectrum" to indicate the ensemble mean escape field, $\langle f\rangle$, as a function of loading rate, $\dot{f} \equiv d f / d t$.

The standard theories of forced barrier crossing can be subdivided into two classes: phenomenological and model-based. The phenomenological hypothesis has been extensively used in studying the failure of solids [10] and in the mechanical rupture of intermolecular bonds [11]. It is assumed that the rate of activated escape takes an Arrhenius form, $k=v_{0} \mathrm{e}^{-\Delta U / k_{B} T}$, where $v_{0}$ is Kramers' attempt frequency [9]. The intrinsic energy barrier of the state, $U_{0}$, taken to be significantly larger than $k_{B} T$ (Boltzmann's constant times the temperature), is reduced proportionally to the applied field as, $\Delta U(f)=U_{0}-f y$, where $y$ is a constant specific to the system under study. This hypothesis was first presented by Tobolsky and Eyring [12] and later by Zhurkov [13] to treat the kinetic failure of materials under an applied stress. Bell [14] refined the theory for ligand-receptor dissociation by imposing that the effective barrier to escape

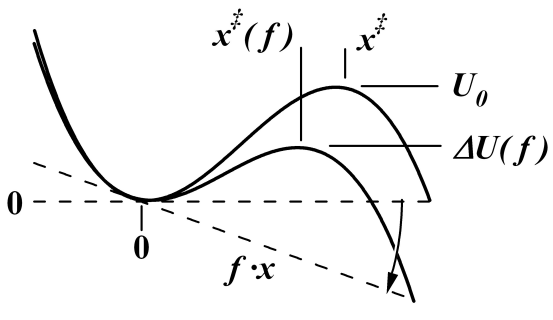

FIG. 1: Schematic of the linear-cubic model energy landscape along the reaction coordinate $x$. When under an increasing force field, $f=\dot{f} t$ (dashed lines), the microscopic details of the barrier follow a time dependence (arrow) which modulates the rate of diffusive passage over the barrier.

should vanish at a critical force given by the ratio of the energy barrier and the distance to the transition state, $f_{c}=U_{0} / x^{\ddagger}$, thus defining the proportionality constant as, $y \equiv x^{\ddagger}$. Hence the phenomenological rate of escape, $k(f)=v_{0} \mathrm{e}^{-U_{0}\left(1-f / f_{c}\right) / k_{B} T}$, often attributed to Bell, has the desired property that when $f=0$ one recovers the intrinsic rate $k_{0}=v_{0} \mathrm{e}^{-U_{0} / k_{B} T}$.

In contrast to the Bell view, model-based theories account for the perturbation of an explicitly defined model potential in the presence of an applied field (Fig. 1). Perhaps the most frequently used model is the linear-cubic potential popularized by Kurkijärvi [15] to study the escape of flux quanta from a superconducting loop. Later Gunther and Barbara [16] and, independently, Garg [7] analyzed a similar model and found asymptotic solutions for the force spectrum at large loading rates. The chief assumption in these approximations is that most escape events occur when the applied field is close to the critical field $\left(f \approx f_{c}\right)$ where the barrier to escape vanishes. Garg's formulism is especially attractive in that it can be extended to a variety of landscape shapes $[17,18]$.

For some time a disconnect existed between solid-state physicists employing the model-based approach and soft condensed-matter physicists using the Bell hypothesis. This changed recently when Dudko et al. [8] showed that Garg's treatment [7] of a linear-cubic potential to study macroscopic quantum tunneling is equally applicable to 
the forced dissociation of intermolecular bonds. It was also shown [17] that for systems driven quickly away from equilibrium, the force spectrum arising from the Bell theory is largely inaccurate compared to the model-based spectrum. This result called into question the utility of the Bell rate in studying forced escape processes. However, a short time later Lin et al. [18] showed that, under a small constant force, any escape rate should reduce to the Bell rate, whereas at large forces a model-based rate is required.

The findings of Lin et al. [18] for systems under a constant load lead to a number of important issues for systems under dynamic loading. Their analysis suggests that the dynamic spectra derived from either the Bell or model-based theories are not competing models, but are actually complements of the complete spectrum. Thus one requires at least two solutions: one for slow $\dot{f}$ based on the Bell theory, and another for fast $\dot{f}$ based on a model-based theory. Since there is no clear definition of when either theory should be used, one must pre-suppose which regime the data are acquired in. Ultimately, to alleviate uncertainty in data fitting and modeling, a force spectrum that is accurate for arbitrary loading rates is desirable.

To address the issues raised above, we now aim to derive the complete dynamic force spectrum. Consider a Brownian particle diffusing within a potential until overcoming an energy barrier to escape. When the particle damping coefficient, $\eta$, is large, the spatial density of states, $W(x, t)$, of an ensemble of such particles is governed by Smoluchowski's equation of motion $\partial_{t} W(x, t)=\frac{k_{B} T}{\eta} \partial_{x}\left(\partial_{x}+\frac{1}{k_{B} T} \partial_{x} V(x, t)\right) W(x, t)$. Here the potential $V(x, t)=U(x)-f x$ is the combination of the intrinsic energy landscape, $U(x)$, and the bias due to the applied force field, $f x$ (see Fig. 1). It is assumed configurational relaxation of the system occurs over a timescale much smaller than the measurement timescale. We further assume the process to be adiabatic, that is, dissipation of energy due to viscous drag is negligible. This is justified by considering a parabolic intrinsic potential, $U(x)=\frac{1}{2} k_{m} x^{2}$, of typical bond stiffness $k_{m}=10 \mathrm{~N} / \mathrm{m}$. The combined potential including the applied force can be expressed as $V(x)=\frac{1}{2} k_{m}(x-u t)^{2}-f^{2} /\left(2 k_{m}\right)$, where $u=\dot{f} / k_{m}$ is the velocity of the potential minimum. In this example, the mean position of $W(x, t)$ lags behind the equivalent quasi-static (canonical) distribution by $\delta x=\eta u / k_{m}$ [19]. Even under a fast loading rate of $\dot{f}=100 \mu \mathrm{N} / \mathrm{s}$, and reasonable damping of $\eta=10^{-8} \mathrm{~kg} / \mathrm{s}$, this gives a clearly negligible lag of only $\delta x=10 \mathrm{fm}$. Therefore, we treat the density of states within the potential as quasi-static, and invoke Kramers' solution [9] of Smoluchowski transport over a barrier at each instance in time. Kramers' escape rate for various model energy landscapes are given elsewhere [20]. Here we begin by considering a fairly generalized escape rate in the pres- ence of a force field [7],

$$
k(f)=v_{0}\left(1-f / f_{c}\right)^{b-1} \mathrm{e}^{-U_{0}\left(1-f / f_{c}\right)^{b} / k_{B} T},
$$

where $f_{c}=b U_{0} / x^{\ddagger}$ is the critical field at which the barrier to escape vanishes. The parameter $b$ selects the particular model of the potential energy well: $b=3 / 2$ models a linear-cubic potential [7] (see Fig. 1), $b=2$ models a harmonic potential, and $b=1$ recovers the phenomenological theory [17]. In the context of field induced switching in magnetic materials, $b=2$ is used when the applied field is parallel with the anisotropy vector, whereas $b=3 / 2$ for more general orientations [21]. Qualitatively, the harmonic potential idealizes the normally anharmonic form of most physical potentials. Therefore, in most cases the asymmetric model given by $b=3 / 2$ is the appropriate choice.

For each observation it is assumed that the field is increased until a single escape event occurs. Thus we assume barrier re-crossing is suppressed by experimental circumstances, such as the directionality of the applied field. This scenario is described by a first order rate process, $d p(t) / d t=-k(t) p(t)$, where $p(t)$ is the probability escape has not occurred up to a time $t$. It is convenient to convert this process from a time to force dependence $(\dot{f}=d f / d t)$,

$$
\int_{1}^{p} \frac{d p^{\prime}}{p^{\prime}}=-\frac{1}{\dot{f}} \int_{0}^{f} k\left(f^{\prime}\right) d f^{\prime} .
$$

Using Eq. (1) in Eq. (2) yields the inverse of the cumulative distribution function of escape fields $\left(p^{-1} \equiv f(p)\right)$,

$$
f(p)=f_{c}\left\{1-\left[1-\frac{k_{B} T}{U_{0}} \ln \left(1-\frac{\ln p}{X}\right)\right]^{1 / b}\right\},
$$

where,

$$
X \equiv \frac{v_{0} \mathrm{e}^{-U_{0} / k_{B} T} f_{c} k_{B} T}{\dot{f} b U_{0}}=\frac{k_{0} k_{B} T}{\dot{f} x^{\ddagger}} .
$$

The mean escape field is then given by $\langle f\rangle=\int_{0}^{1} f(p) d p$, which cannot be carried out analytically. To avoid expanding near a particular loading regime we take the next significant inner function, $\alpha \equiv \ln (1-\ln p / X)$, and treat $f$ as a function of the new random variable $\alpha$. Averaging over the expansion of $f(\alpha)$ around $\langle\alpha\rangle$ we find, $\langle f\rangle=f(\langle\alpha\rangle)+\frac{1}{2} f^{\prime \prime}(\langle\alpha\rangle) \sigma_{\alpha}^{2}+\cdots$. When $b=1$ we have $\langle f\rangle=f(\langle\alpha\rangle)$, and for $b$ close to unity the second and higher derivatives of $f(\alpha)$ contribute negligibly to the mean. Given that most models of forced barrier crossing will have $1 \leq b \leq 2$ (see discussion on $b$ above), the higher order terms in $\langle f\rangle$ can be neglected in favor of the leading term $f(\langle\alpha\rangle)$. Evaluating $\langle\alpha\rangle=\int_{0}^{1} \alpha(p) d p$, we find the complete mean escape field is given by the transcendental equation, 


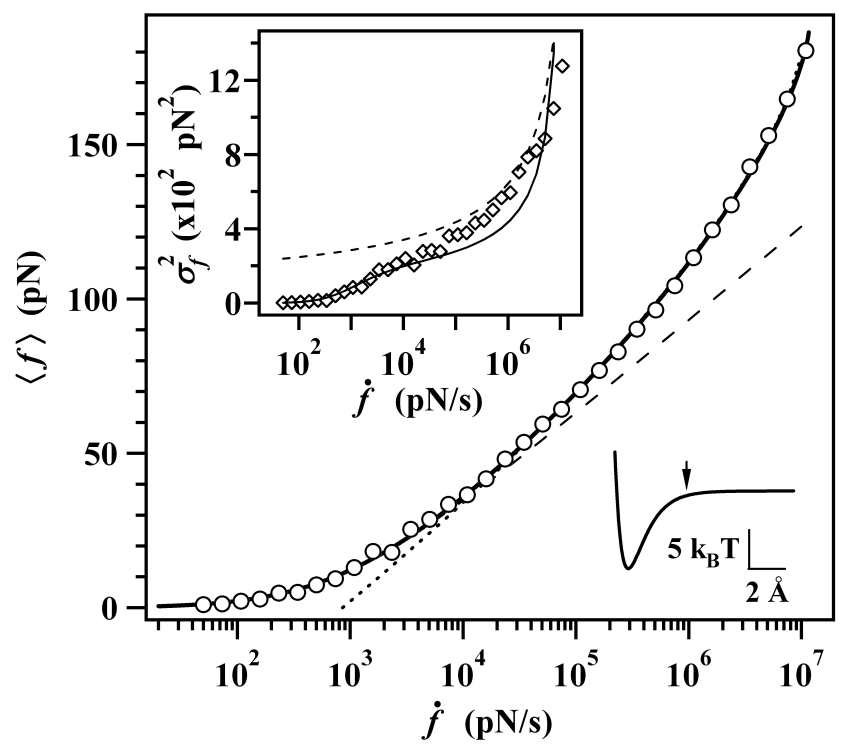

FIG. 2: Non-linear least-squares fit of Eq. (5) (solid curve passing behind points), with $b=3 / 2$, against Brownian dynamics simulations of forced escape (open circles) from a Morse potential (drawn in the lower inset). Fit results give $U_{0}=9.49 \pm 0.04 k_{B} T, x^{\ddagger}=3.14 \pm 0.03 \AA$ (arrow), and $k_{0}=36.45 \pm 1.41 \mathrm{~s}^{-1}$. From simulations at zero force the intrinsic rate is found to be $k_{0}=37.06 \mathrm{~s}^{-1}$. Equations (7) (dashed curve, converging at low $\dot{f}$ ) and (8) (dotted curve, converging at high $\dot{f}$ ) are shown for comparison. Upper inset displays the variance in the simulated escape forces (diamonds) compared to Eq. (6) (solid curve), and the high-force approximation (dashed curve, see text), calculated at the fitted parameter values.

$$
\langle f\rangle \cong f_{c}\left\{1-\left[1-\frac{k_{B} T}{U_{0}} \mathrm{e}^{X} E_{1}(X)\right]^{1 / b}\right\},
$$

where $E_{1}(u)=\int_{u}^{\infty} \frac{\mathrm{e}^{-s}}{s} d s$ is the exponential integral. Equation (5) is a complete dynamic force spectrum for single barrier potentials, and is the main result of this Letter. Note that using $\mathrm{e}^{X} E_{1}(X) \approx \ln \left(1+\mathrm{e}^{-\gamma} / X\right)$, where $\gamma=0.577 \ldots$ is the Euler constant, gives a useful interpolation to Eq. (5). Likewise, the variance in the escape field distribution, $\sigma_{f}^{2}=\left\langle f^{2}\right\rangle-\langle f\rangle^{2}$, can be estimated by $\sigma_{f}^{2} \cong f^{\prime}(\langle\alpha\rangle)^{2} \sigma_{\alpha}^{2}$. Expanding $\alpha(p)$ around the (constant rate) mean probability, $p=1 / e$, gives to leading order, $\sigma_{\alpha}^{2} \sim 1 /(1+X)^{2}$, and the variance follows as,

$$
\sigma_{f}^{2} \cong\left[\frac{k_{B} T}{x^{\ddagger}(1+X)}\right]^{2}\left[1-\frac{k_{B} T}{U_{0}} \mathrm{e}^{X} E_{1}(X)\right]^{2 / b-2} .
$$

The performance of Eqs. (5) and (6) are demonstrated in Fig. 2 by comparison against Brownian dynamics simulations. While Eq. (6) is asymptotically correct at both high and low $\dot{f}$, it underestimates the simulated data for intermediate loading rates. A high-force approximation for the variance has been found previously [7, 17], $\sigma_{f}^{2} \cong \frac{1}{6}\left[\pi k_{B} T / x^{\ddagger}\right]^{2}\left[\frac{k_{B} T}{U_{0}} \ln \left(\mathrm{Xe}^{1+\mathrm{U}_{0} / \mathrm{k}_{\mathrm{B}} \mathrm{T}}\right)\right]^{2 / b-2}$, which follows the data well at intermediate and large loading rates (see Fig. 2 inset).

The familiar models used for studying forced barrier crossing are special cases of the complete spectrum presented here. For example, when the loading rate is very small $(\dot{f} \sim 0)$, or when the barrier to rupture is very large $\left(k_{B} T / U_{0} \sim 0\right)$, Eq. (5) reduces to,

$$
\langle f\rangle \approx \frac{k_{B} T}{x^{\ddagger}} \mathrm{e}^{X} E_{1}(X)+\cdots .
$$

Equation (7) is the exact result retrieved from evaluating the mean escape field under the Bell escape rate [22]. Furthermore, by taking the large $\dot{f}$ limit of Eq. (7) we find the model frequently used for analyzing single molecule bond rupture data $[5,6,11,22],\langle f\rangle \approx \frac{k_{B} T}{x^{\ddagger}} \ln \left(\mathrm{e}^{-\gamma} / X\right)$. Therefore, all of the simple model landscapes (defined by b) converge to a universal spectrum given by the phenomenological Bell theory. Such general behavior can be reconciled by considering that for slow loading rates the shape of an underlying landscape is minimally perturbed before the average escape event occurs [18]. Thus the small lowering of the activation barrier is the primary contributor to the escape dynamics. Given that Eq. (7) arises from a minimal description of the underlying potential (contained in $x^{\ddagger}$ ), the practical significance of this result is that the parameters of Eq. (7), for small loading rates, are independent of the landscape shape. The fact that the phenomenological result describes the entire spectrum in the limit $U_{0} \rightarrow \infty$ implies that larger barriers extend the range of validity of Eq. (7). This may explain why a vast amount of experimental investigations $[5,6,10,11,13,22,23]$ have matched well against the phenomenological theory. This also underscores the importance of sampling $\dot{f}$ over several orders of magnitude, otherwise fitting Eq. (5) to experimental data may result in an ambiguous value for $U_{0}$.

Alternatively, taking a direct expansion of Eq. (5) in the large $\dot{f}$ regime $(X \sim 0)$ leads to,

$$
\langle f\rangle \approx f_{c}\left\{1-\left[1-\frac{k_{B} T}{U_{0}} \ln \frac{\mathrm{e}^{-\gamma}}{X}\right]^{1 / b}\right\}+\cdots,
$$

which is the asymptotic result found previously [7] for general over-damped barrier crossings. Equation (8), and similar approximations, have been explored in the context of field switching in domain-wall junctions [16], magnetic nanoparticles [1, 4], and the rupture of single molecule bonds [8, 17, 18, 24]. Eq. (8) is accurate when systems are driven quickly away from equilibrium, but deviates for small $\dot{f}$, and becomes negative when $\dot{f} \lesssim k_{0} k_{B} T / x^{\ddagger}$. Experimental deviations from Eq. (8) for small $\dot{f}$ should not be considered anomalous, and can be modeled appropriately using Eq. (5).

Finally, the model presented here is applied to experimental data of forced barrier crossing from two different 

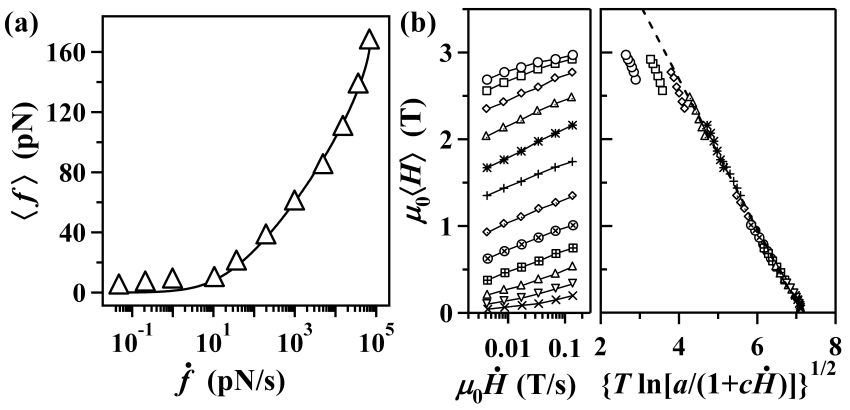

FIG. 3: (a) Fit of Eq. (5) (solid curve, $b=3 / 2$ ) to biotin-avidin bond rupture data $(\triangle)$ reproduced with permission from [23], copyright 1999 by Macmillan Publishers Ltd: Nature. (b) Mean switching field $\langle H\rangle$ versus sweeprate $(\dot{H} \equiv d H / d t)$ for a single molecule magnet at temperatures from $0.4 \mathrm{~K}$ (upper most spectrum, O) to $2.6 \mathrm{~K}$ (bottom most spectrum, $\times$ ), in $0.2 \mathrm{~K}$ increments; reproduced with permission from [1], copyright 2005 by the American Physical Society. Globally fitting to these spectra yields $v_{0}=1.21 \pm 0.31 \times 10^{8} \mathrm{~s}^{-1}, \mu_{0} H_{c}=6.15 \pm 0.06 \mathrm{~T}$, and $U_{0} / k_{B}=50.65 \pm 0.68 \mathrm{~K}$. Using the fitted parameters, the right panel in (b) shows scaling of the data onto a single master curve (see text) for temperatures above $0.8 \mathrm{~K}$.

systems. Shown in Fig. 3(a) is the dynamic rupture spectrum of the biotin-avidin bond reproduced from Ref. [23]. Equation (5) fits remarkably well over the more than million-fold range of loading rates explored. The fitted energy barrier height of $U_{0}=4.93 \pm 0.23 \mathrm{kcal} / \mathrm{mol}$ and transition state of $x^{\ddagger}=3.07 \pm 0.22 \AA$ matches very well with the energy landscape rigorously reconstructed from the same data [25]. Figure 3(b), shows the low temperature magnetization reversal of a single-chain magnet taken from Ref. [1]. The spectra at temperatures above $T=0.8 \mathrm{~K}$ were globally fit to Eq. (5), with $b=2$. The data are then plotted as the mean switching field against $\{T \ln [a /(1+c \dot{H})]\}^{1 / 2}$, with $a=e^{U_{0} / k_{B} T}$ and $c=e^{-\gamma} b U_{0} /\left(v_{0} H_{c} k_{B} T e^{-U_{0} / k_{B} T}\right)$, where we have used the interpolative approximation to Eq. (5). This results in a linear scaling of the data for both the intermediate and high temperature data (right panel of Fig. 3(b)). As expected, the data taken below $1 \mathrm{~K}$ deviate from the scaling due to quantum tunneling [1].

In summary, the primary result of this work provides a complete dynamic force spectrum (Eq. (5)). The solution bridges two prominent approximations which were recently predicted to represent limiting regimes. This result explicitly demonstrates that for single-barrier states loaded slowly by an external field, the force spectrum can be universally modeled by the phenomenological Bell hypothesis. Equation (3) may find use in monte carlo modeling of complex systems, such as protein unfolding [26], where individual transitions can be pseudo-randomly selected by inverse transform sampling [27]. The theory presented here is general to a broad range of condensed- matter and materials science studies of dynamicallyforced, thermally activated transitions in single-molecule systems.

The author thanks S. Roger Qiu, Jonathan R.I. Lee, Aleksandr Noy, and Donald Sirbuly for helpful comments and Wolfgang Wernsdorfer for pointing out relevant literature. This work was performed under the auspices of the U.S. Department of Energy by Lawrence Livermore National Laboratory under Contract DE-AC52-07NA27344. UCRL-JRNL-232061.

[1] W. Wernsdorfer, R. Clérac, C. Coulon, L. Lecren, and H. Miyasaka, Phys. Rev. Lett. 95, 237203 (2005).

[2] S. B. Smith, Y. Cui, and C. Bustamante, Meth. Enzym. 361, 134 (2003).

[3] C. Ke, M. Humeniuk, H. S-Gracz, and P. E. Marszalek, Phys. Rev. Lett. 99, 018302 (2007).

[4] W. Wernsdorfer, E. B. Orozco, K. Hasselbach, A. Benoit, B. Barbara, N. Demoncy, A. Loiseau, H. Pascard, and D. Mailly, Phys. Rev. Lett. 78, 1791 (1997).

[5] T. A. Sulchek, R. W. Friddle, K. Langry, E. Lau, H. Albrecht, T. V. Ratto, S. DeNardo, M. Colvin, and A. Noy, Proc. Nat. Acad. Sci. 102, 16638 (2005).

[6] E. Evans and K. Ritchie, Biophys. J. 72, 1541 (1997).

[7] A. Garg, Phys. Rev. B 51 (1995).

[8] O. K. Dudko, A. E. Filippov, J. Klafter, and M. Urbakh, Proc. Nat. Acad. Sci. 100, 11378 (2003).

[9] H. A. Kramers, Physica 7, 284 (1940).

[10] See for example D. R. Curran and L. Seaman and D. A. Shockey, Phys. Rep. 147, 253 (1987).

[11] For a recent review see E. Evans and D. A. Calderwood, Science 316, 1148 (2007).

[12] A. Tobolsky and H. Eyring, J. Chem. Phys. 11, 125 (1943).

[13] S. N. Zhurkov, Int. J. Fract. Mech. 1, 311 (1965).

[14] G. I. Bell, Science 200, 618 (1978).

[15] J. Kurkijärvi, Phys. Rev. B 6, 832 (1972).

[16] L. Gunther and B. Barbara, Phys. Rev. B 49, 3926 (1994).

[17] O. Dudko, G. Hummer, and A. Szabo, Phys. Rev. Lett. 96 (2006).

[18] H.-J. Lin, H.-Y. Chen, Y.-J. Sheng, and H.-K. Tsao, Phys. Rev. Lett. 98 (2007).

[19] O. Mazonka and C. Jarzynski, cond-mat p. 9912121 (1999).

[20] P. Hänggi, P. Talkner, and M. Borkovec, Rev. Mod. Phys. 62, 251 (1990).

[21] R. H. Victora, Phys. Rev. Lett. 63, 457 (1989).

[22] C. Gergely, J.-C., P. Schaaf, B. Senger, M. Maaloum, J. K. H. Hörber, and J. Hemmerlé, Proc. Nat. Acad. Sci. 97, 10802 (2000).

[23] R. Merkel, P. Nassoy, A. Leung, K. Ritchie, and E. Evans, Nature 397, 50 (1999).

[24] Y.-J. Sheng, S. Jiang, and H.-K. Tsao, J. Chem. Phys. 123, 091102 (2005).

[25] B. Heymann and H. Grubmüller, Phys. Rev. Lett. 84, 6126 (2000).

[26] A. Imparato, A. Pelizzola, and M. Zamparo, Phys. Rev. Lett. 98, 148102 (2007). 
[27] L. Devroye, Non-uniform random variate generation (New York: Springer-Verlag, 1986). 